Supporting Information for:

\title{
Tunable Disassembly of Micelles Using a Redox Trigger
}

\author{
Suhrit Ghosh, Katharine Irvine and S. Thayumanavan* \\ Department of Chemistry, University of Massachusetts, Amherst,
}

Massachusetts 01003

\section{Materials and methods:}

All chemicals and solvents were purchased from commercial sources and were used as such unless otherwise mentioned. ${ }^{1} \mathrm{H}$ NMR spectra were recorded on a $400 \mathrm{MHz}$ Bruker NMR spectrometer using the residual proton resonance of the solvent as the internal standard. Chemical shifts are reported in parts per million (ppm). When peak multiplicities are given, the following abbreviations are used: s, singlet; d, doublet; $t$, triplet $\mathrm{m}$, multiplate. ${ }^{13} \mathrm{C}$-NMR spectra were proton decoupled and recorded on a 100 $\mathrm{MHz}$ Bruker spectrometer using carbon signal of the deuterated solvent as the internal standard. EI (electron impact) mass spectra were obtained using JEOL MStation JMS700 high-resolution two-sector spectrometer. Elemental analysis was performed in PerkinElmer 2400 Series II CHN instrument. Flash column chromatography was performed using 37-75 $\mu \mathrm{M}$ silica gel.

\section{Synthesis:}

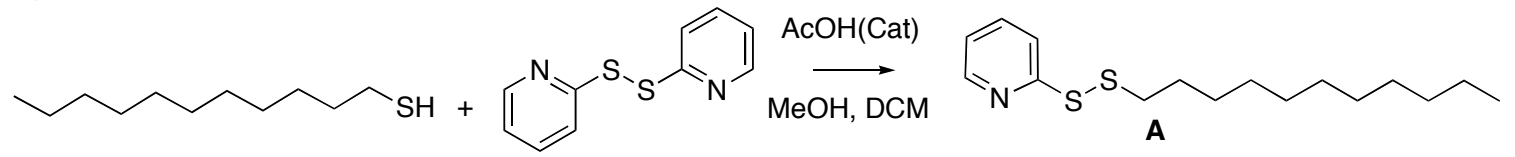

Compound A: $2.0 \mathrm{~g}(9.1 \mathrm{mmol})$ of 2,2' pyridine disulfide (aldrithiol) was dissolved in 12 $\mathrm{mL}$ of methanol and was added with $134 \mu \mathrm{L}$ of glacial acetic acid. To this mixture, a solution of undecanethiol $(1.0 \mathrm{~mL}, 4.5 \mathrm{mmol})$ in $5 \mathrm{~mL}$ dichloromethane was added dropwise from an addition funnel. Once the addition was over the reaction mixture was stirred at room temperature for additional $3 \mathrm{~h}$. The stirring was stopped, solvent was evaporated in the rotary evaporator and the crude product was purified by flash column chromatography using silica gel as stationary phase and mixture of ethyl acetate and hexane (1:9 v/v ratio) as eluent. $0.95 \mathrm{~g}$ pure product was obtained as a light greenish oil in $68 \%$ yield.

${ }^{1} \mathrm{H}$ NMR $\left(\mathrm{CDCl}_{3}\right): \delta(\mathrm{ppm}): 8.46(\mathrm{~d}, 1 \mathrm{H}), 7.72(\mathrm{~m}, 1 \mathrm{H}), 7.63(\mathrm{~m}, 1 \mathrm{H}), 7.00(\mathrm{~m}, 1 \mathrm{H}), 2.8$ $(\mathrm{t}, 2 \mathrm{H}), 1.7(\mathrm{~m}, 2 \mathrm{H}), 1.40-1.19(\mathrm{~m}, 16 \mathrm{H}), 0.9(\mathrm{t}, 3 \mathrm{H})$

${ }^{13} \mathrm{C} \mathrm{NMR}\left(\mathrm{CDCl}_{3}\right): \delta(\mathrm{ppm}): 160.7,149.4,136.8,120.3,119.4,39.0,31.9,29.58-28.5$, 22.6,14.1

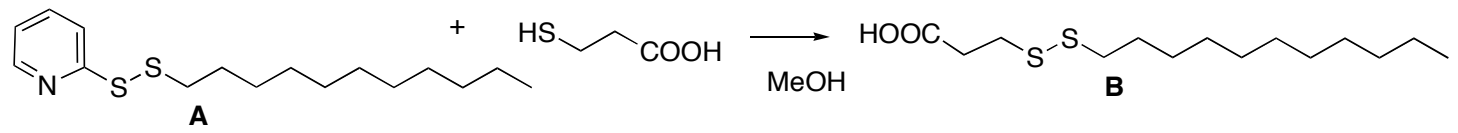

Compound B (acid form of surfactant 1): $0.8 \mathrm{~g}(2.6 \mathrm{mmol}$ ) of compound A, $234 \mu \mathrm{L}$ (2.6 mmol) thiopropionic acid, $79 \mu \mathrm{L}$ glacial acetic acid was dissolved in $10 \mathrm{~mL}$ methanol and 
the reaction mixture was stirred at room temperature for $3 \mathrm{~h}$. The reaction was stopped and the solvent was evaporated. The crude product was purified by flash column chromatography using silica gel as stationary phase and mixture of ethyl acetate/hexane $(2: 98 \mathrm{v} / \mathrm{v} \mathrm{ratio})$ as eluent. $0.7 \mathrm{~g}$ of the pure product was obtained as white solid in $93 \%$ yield.

${ }^{1} \mathrm{H} \mathrm{NMR}\left(\mathrm{CDCl}_{3}\right): \delta(\mathrm{ppm}): 2.91(\mathrm{t}, 2 \mathrm{H}), 2.83(\mathrm{t}, 2 \mathrm{H}), 2.68(\mathrm{t}, 2 \mathrm{H}), 1.62(\mathrm{~m}, 2 \mathrm{H})$, $1.41-1.20(\mathrm{~m}, 16 \mathrm{H}), 0.89(\mathrm{t}, 3 \mathrm{H})$

${ }^{13} \mathrm{C} \mathrm{NMR}\left(\mathrm{CDCl}_{3}\right): \delta(\mathrm{ppm}): 187.2,39.0,34.0,32.7,31.9,29.6-29.29,28.5,22.7,14.1$.

Mass spectra (EI) data: $\mathrm{m} / \mathrm{z} 292.12$ (Calculated for $\mathrm{C}_{14} \mathrm{H}_{28} \mathrm{O}_{2} \mathrm{~S}_{2}$ 292.15). Elemental analysis data: Calcd for $\mathrm{C}_{14} \mathrm{H}_{28} \mathrm{O}_{2} \mathrm{~S}_{2}$ : C, 57.49; H, 9.65; N, 0.0. Found C, 57.64; H, 9.74; N, 0.02 All values are given as percentages. M.p.: Decomposed before melting at $73{ }^{\circ} \mathrm{C}$.

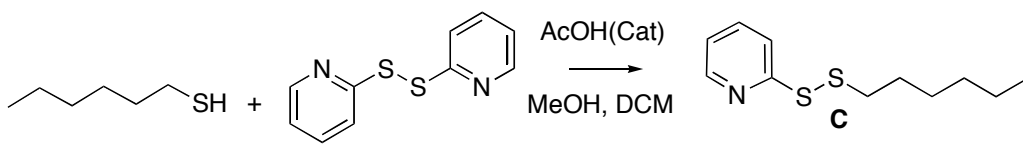

Compound $\mathbf{C}$ : Compound $\mathrm{C}$ was prepared following exactly the procedure as described for compound A. Instead of 1-undecanethiol, in this case 1-hexanethiol was used. Yield: $71 \%$. The product was obtained as light yellow liquid.

${ }^{1} \mathrm{H}$ NMR $\left(\mathrm{CDCl}_{3}\right): \delta(\mathrm{ppm}): 8.45(\mathrm{~d}, 1 \mathrm{H}), 7.72(\mathrm{~m}, 1 \mathrm{H}), 7.62(\mathrm{~m}, 1 \mathrm{H}), 7.08(\mathrm{~m}, 1 \mathrm{H}), 2.79$ $(\mathrm{t}, 2 \mathrm{H}), 1.72(\mathrm{~m}, 2 \mathrm{H}), 1.42-1.21(\mathrm{~m}, 6 \mathrm{H}), 0.91(\mathrm{t}, 3 \mathrm{H})$

${ }^{13} \mathrm{C}$ NMR $\left(\mathrm{CDCl}_{3}\right): \delta(\mathrm{ppm}): 158.7,149.1,137.8,121.5,119.8,39.0,31.9,29.6,27.1$, $22.8,14.1$

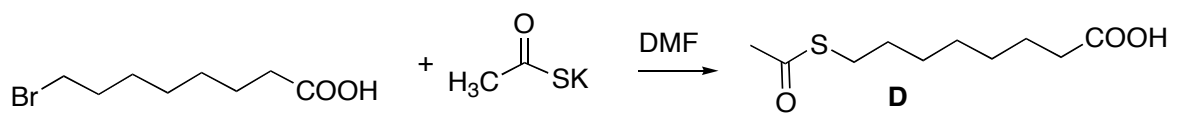

Compound D: 7-bromoheptanoic acid, (1.84 g, $8.25 \mathrm{mmol})$ and potassium thioacetate $(1.13 \mathrm{~g})$ were dissolved in $10 \mathrm{~mL}$ DMF and the mixture was stirred under argon atmosphere at $60{ }^{\circ} \mathrm{C}$ for $8 \mathrm{~h}$. The heating was stopped, the reaction mixture was cooled to room temperature and poured in $50 \mathrm{~mL}$ ice-cold water. The product was extracted by $(3 \mathrm{x}$ 30) $\mathrm{mL}$ diethyl ether. The combined organic layer was washed with $(2 \times 20) \mathrm{mL}$ water and then dried over anhydrous $\mathrm{Na}_{2} \mathrm{SO}_{4}$. The solvent was evaporated to get the crude product as light brown color liquid. The ${ }^{1} \mathrm{H}$ NMR was taken and it was found to be the only product. The product was not purified at this stage and was taken to the next step as such. Yield of the crude product : $94 \%$

${ }^{1} \mathrm{H}$ NMR $\left(\mathrm{CDCl}_{3}\right): \delta(\mathrm{ppm}): 2.86(\mathrm{t}, 2 \mathrm{H}), 2.36-2.30(\mathrm{~m}, 5 \mathrm{H}), 1.64-1.33(\mathrm{~m}, 10 \mathrm{H})$

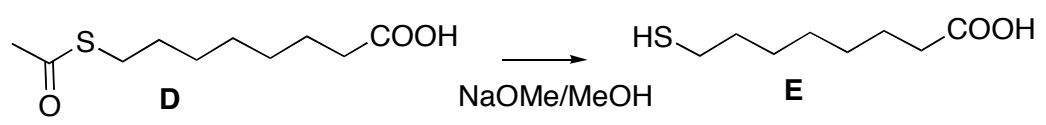

Compound E: Freshly cut sodium $(0.5 \mathrm{~g}, 2.17 \mathrm{mmol})$ was added to $10 \mathrm{~mL}$ dry $\mathrm{MeOH}$ and the solution was stirred under argon atmosphere for 15 minutes. Compound D (1.8 g, 7.4 
mmol) was added to the solution and the mixture was stirred at room temperature for $8 \mathrm{~h}$. The reaction was stopped, was cooled in an ice bath and was acidified with conc. $\mathrm{HCl}$. The solvent was evaporated and the solution was added with $20 \mathrm{~mL}$ ether, it was washed with $(2 \times 20) \mathrm{mL}$ distilled water. The combined organic layer was dried over $\mathrm{Na}_{2} \mathrm{SO}_{4}$ and concentrated to get the crude product as light yellow oil in $89 \%$ yield. ${ }^{1} \mathrm{H}$ NMR was checked and found the compound is reasonably pure. It was taken to the next step without further purification.

${ }^{1} \mathrm{H}$ NMR $\left(\mathrm{CDCl}_{3}\right): \delta(\mathrm{ppm}): 2.66(\mathrm{t}, 2 \mathrm{H}), 2.34(\mathrm{t}, 2 \mathrm{H}), 1.67-1.58(\mathrm{~m}, 4 \mathrm{H}), 1.41-1.32(\mathrm{~m}$, $6 \mathrm{H})$.

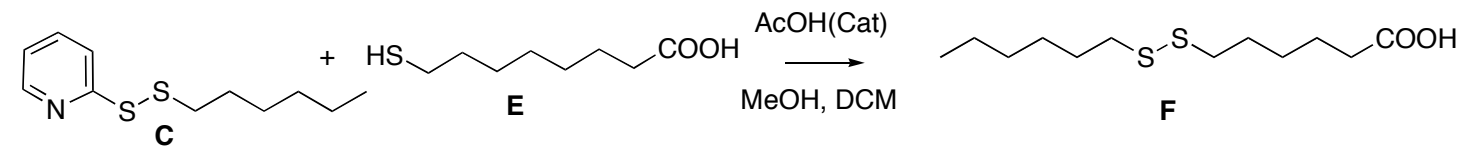

Compound F: Compound $\mathrm{F}$ was prepared compound $\mathrm{C}$ and $\mathrm{E}$, following exactly the similar procedure as described for compound B. Yield: $91 \%, \mathrm{mp}: 43{ }^{\circ} \mathrm{C}$

${ }^{1} \mathrm{H}$ NMR $\left(\mathrm{CDCl}_{3}\right): \delta(\mathrm{ppm}): 2.91(\mathrm{t}, 2 \mathrm{H}), 2.83(\mathrm{t}, 2 \mathrm{H}), 2.68(\mathrm{t}, 2 \mathrm{H}), 1.62(\mathrm{~m}, 2 \mathrm{H})$,

$1.41-1.20(\mathrm{~m}, 16 \mathrm{H}), 0.89(\mathrm{t}, 3 \mathrm{H})$

${ }^{13} \mathrm{C} \mathrm{NMR}\left(\mathrm{CDCl}_{3}\right): \delta$ (ppm): 187.2, 39.0, 34.0, 32.7, 31.9, 29.6-29.29, 28.5, 22.7, 14.1

Mass spectra (EI) data: $\mathrm{m} / \mathrm{z} 292.15$ (Calcd. for $\mathrm{C}_{14} \mathrm{H}_{28} \mathrm{O}_{2} \mathrm{~S}_{2} 292.15$ )

Elemental analysis data: Calcd. for $\mathrm{C}_{14} \mathrm{H}_{28} \mathrm{O}_{2} \mathrm{~S}_{2}: \mathrm{C}, 57.49 ; \mathrm{H}, 9.65 ; \mathrm{N}, 0.0$. Found $\mathrm{C}$, 57.72; H, 9.51; N, 0.01. All values are given as percentages.

\section{Physical Studies:}

Spectral data for the determination of CMC of Sodium laurate (5).
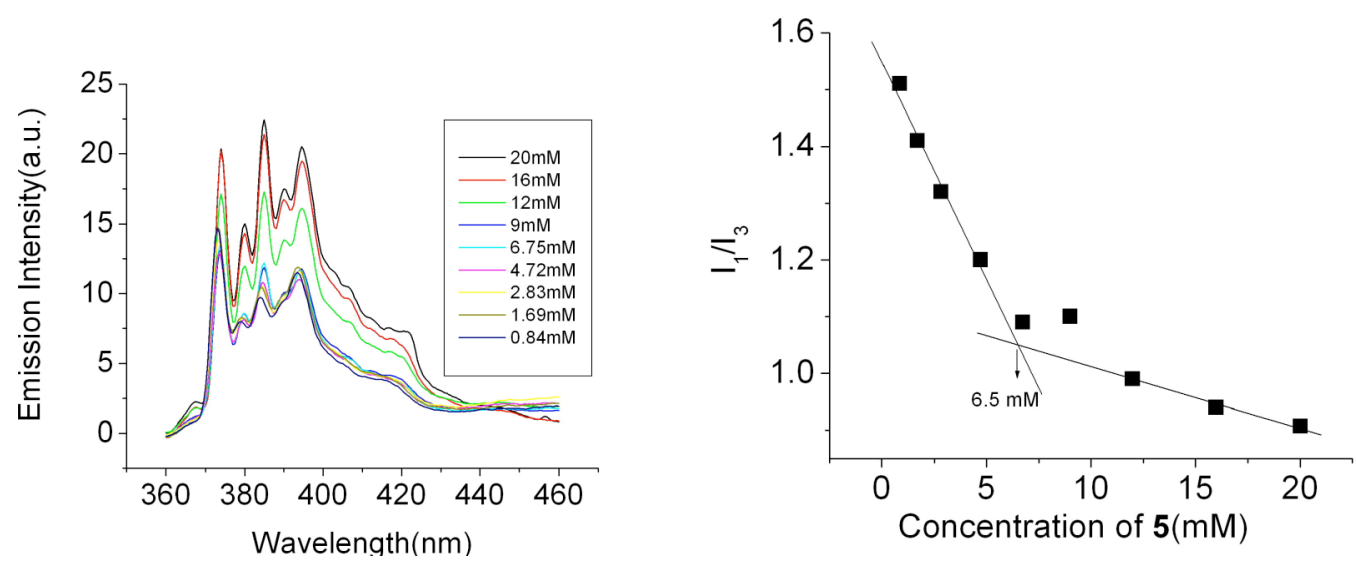

Figure S1: Determination of CMC of sodium laurate (5): left- pyrene emission spectra with varying surfactant concentration (concentration of the surfactant is listed in the legend): rightplot of $I_{1} / I_{3} V s$. the concentration of surfactant. 


\section{Spectral data for determination of CMC of surfactant 1:}
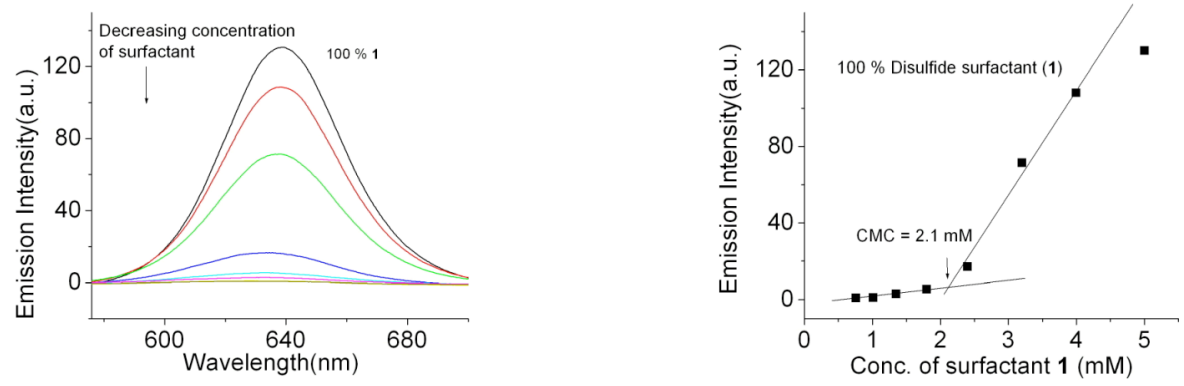

Figure S2: left-Fluorescence spectra of Nile red in various concentration of surfactant 1; rightplot of emission intensity at peak maxima $(637 \mathrm{~nm})$ as a function of concentration of 1

\section{Spectral data for determination of CMC of various mixed micelles}
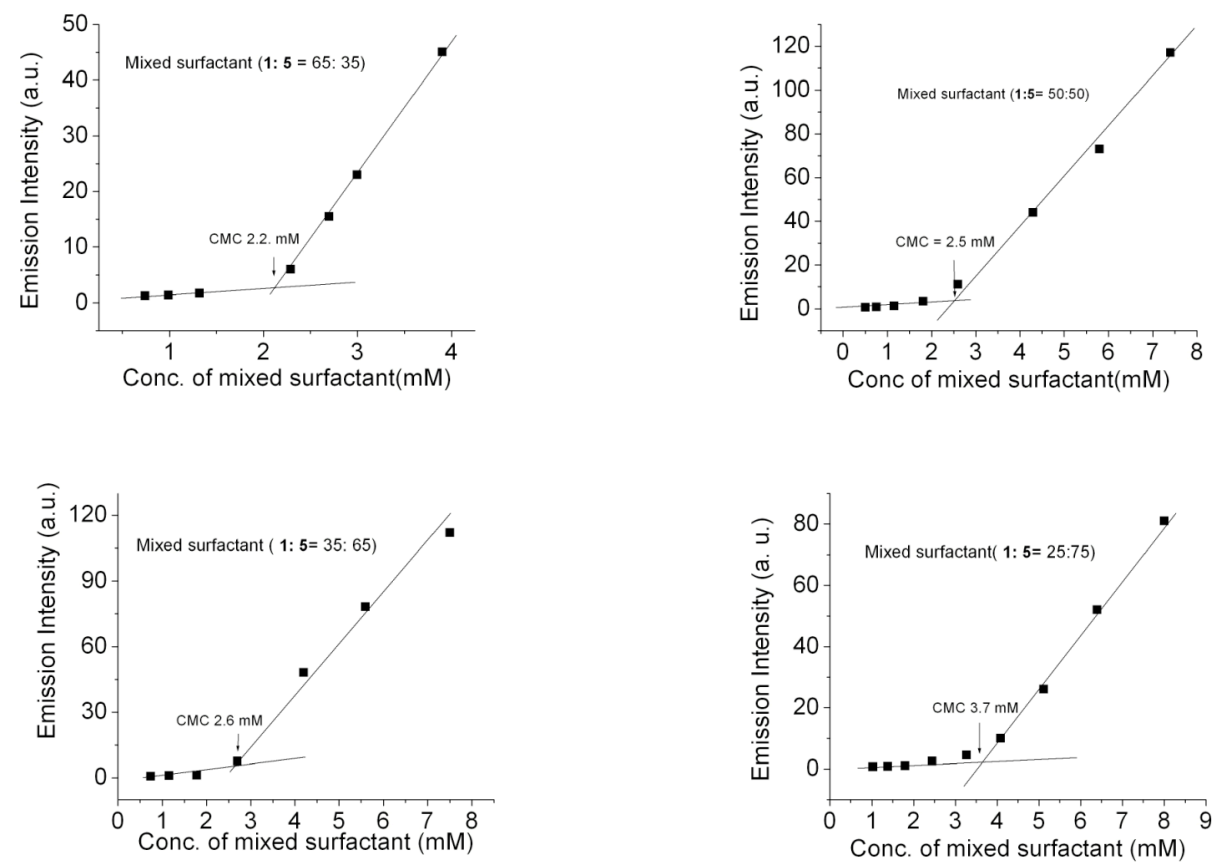

Figure S3: CMC plots for the various mixed micelles formed by surfactants 1 and 5. In each experiment Nile red was used as probe and the initial concentration of it was kept constant at $10^{-5}$ $M$. The ratio of the two surfactants in mixed micelle studied are written inside each plot. In each case the emission intensity of Nile red (conc. $10^{-5} \mathrm{M}$ ) at its emission maximum was plotted against the total concentration of the mixed surfactants. 
Spectral data for DTT induced dye (Nile red) release studies:
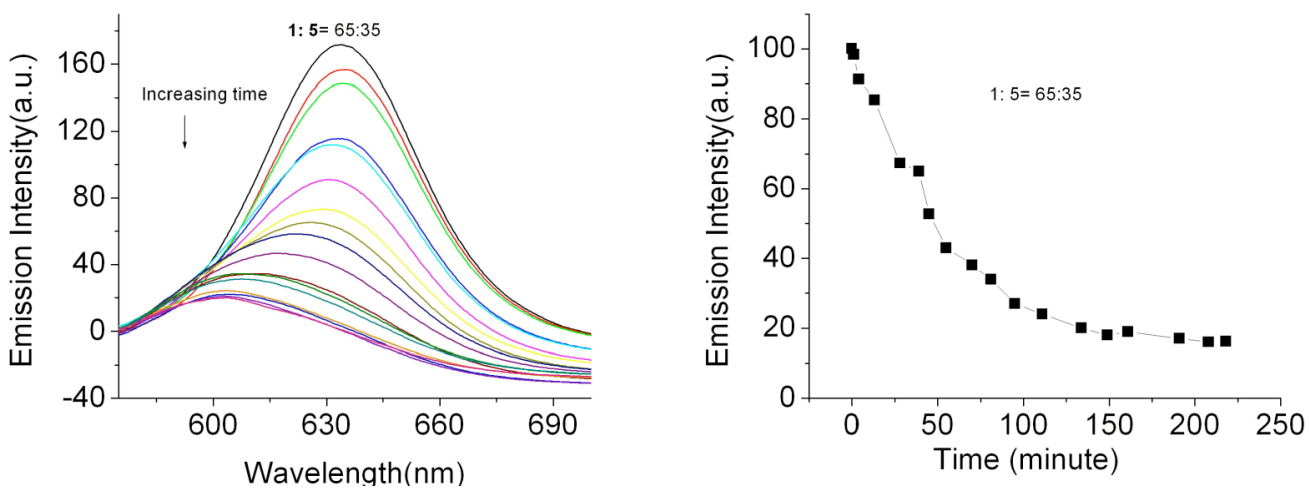

Figure S4: Dye release profile for mixed micelle $(1: 5=65: 35)$; left- the change in fluorescence spectrum with time, right-plot of fluorescence intensity against time. Wave length $(\mathrm{nm})$ at which the intensity is plotted (with increasing time) 633.5, 634, 634, 632.5, 631, $631,630.1,628.73,625,623,617,612,609,607,603,602.5,602,602.5$. Total surfactant concentration $=10 \mathrm{mM}$, Concentration of Nile red encapsulated $=1 * 10^{-5} \mathrm{M}$, and ratio of surfactant 1: $D T T=1: 1$
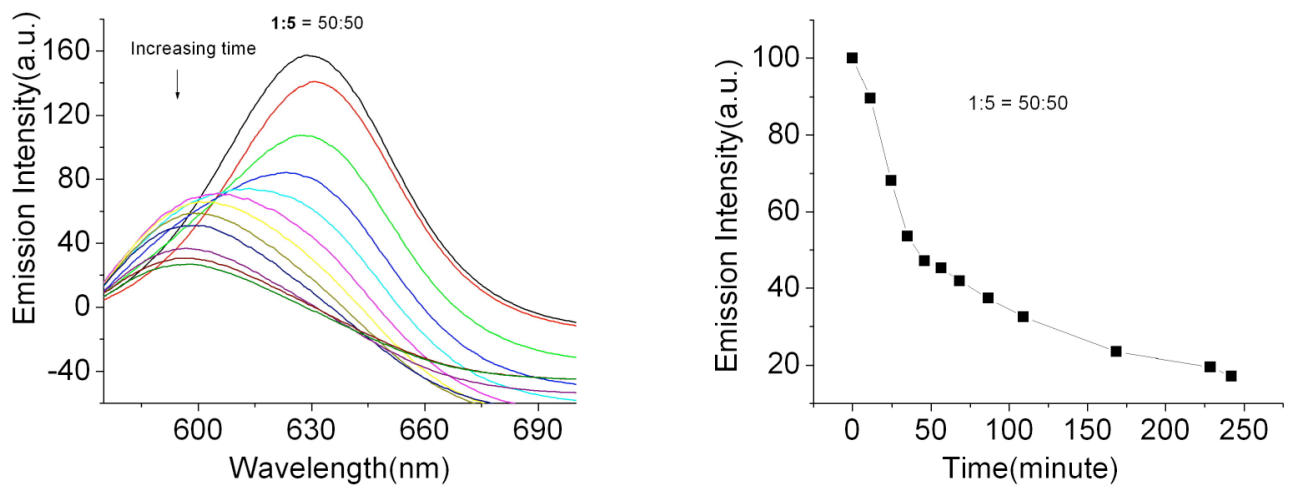

Figure S5: Dye release profile for mixed micelle $(1: 5=50: 50)$; left- the change in fluorescence spectra with time, right- plot of fluorescence intensity against time. Wave length(nm) at which the intensity is plotted (with increasing time) 628.5, 630.5, 629.5, 623, 613.5, 605.5, 600.5, 599, 599, 596.5, 596, 595.5. Total surfactant concentration $=10 \mathrm{mM}$, Concentration of Nile red encapsulated $1 * 10^{-5} \mathrm{M}$, and ratio of surfactant $1:$ DTT $=1: 1$ 

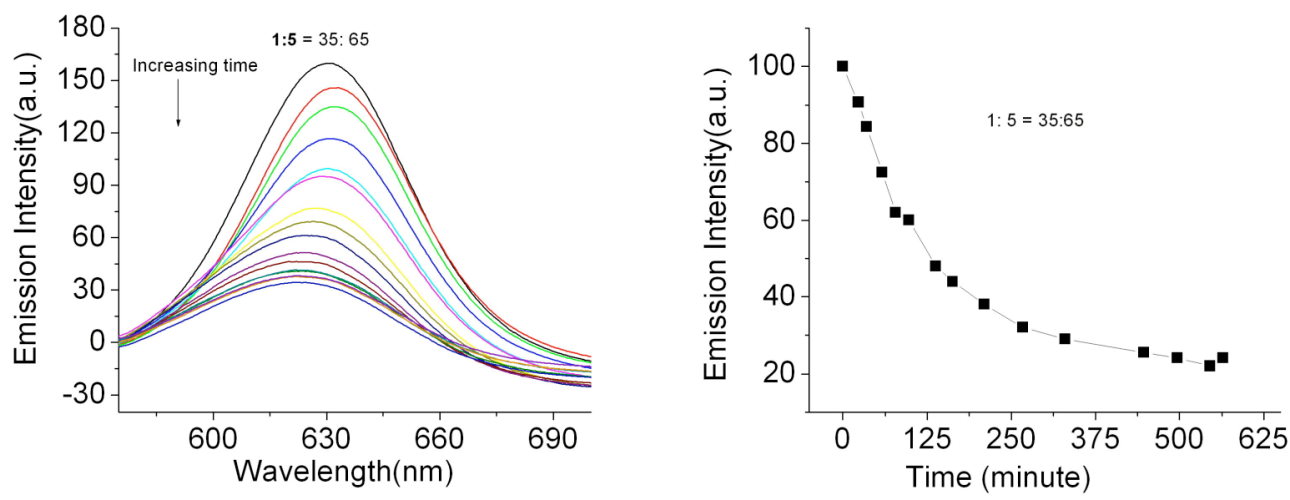

Figure S6: Dye release profile for mixed micelle $(1: 5=35: 65)$; left- the change in fluorescence spectrum with time, right-plot of fluorescence intensity against time; Wave length at which the intensity is plotted (with increasing time) 631, 634, 632, 632, 632, 631, $631.5,630.5,629,628,627.5,624,623.5,622.5,622$. Total surfactant concentration $=10$ $m M$, Concentration of Nile red encapsulated $1 * 10^{-5} \mathrm{M}$, and ratio of surfactant $1: D T T=1: 1$
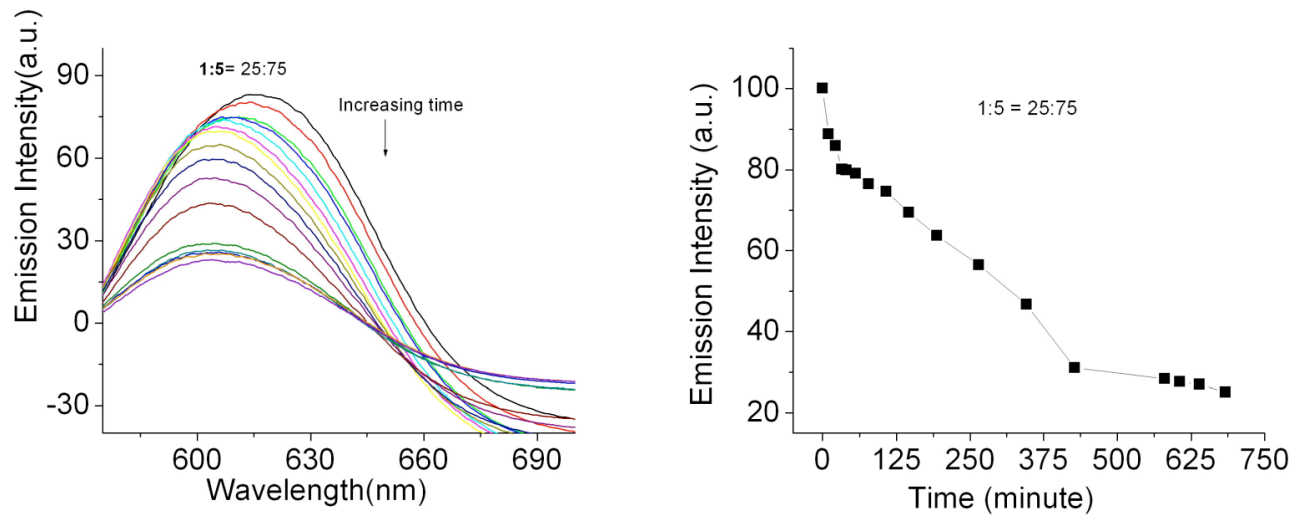

Figure S7: Dye release profile for mixed micelle $(1: 5=25: 75)$; left- the change in fluorescence spectrum with time, right- plot of fluorescence intensity against time: Wave length at which the intensity is plotted (with increasing time) - 621, 614, 614, 611, 609, 607, 606, 606, 605, 604, $604,603,603,602.5,602.0,602$. Total surfactant concentration $=10 \mathrm{mM}$, Concentration of Nile red encapsulated $1 * 10^{-5} \mathrm{M}$, and ratio of surfactant $1: D T T=1: 1$

Note: The emission spectra of Nile red in all the mixed micelle system experiences a blue shift with time. It is known that Nile red emission spectrum is shifted towards lower wavelength with decreasing polarity of the medium (J. Org. Chem. 2006, 7835). It is important to note that Nile Red is not soluble in water on it's own at least to the extent where one can see it's emission spectral signature. Thus the observed emission is from those dye molecules, which are located inside the micelle. So, it's reasonable to state that with increasing time the local environment of the dye molecules is becoming more hydrophobic. When the disulfide surfactants are continuously being cleaved, the composition of the surfactants is the mixed micelle is altered. It is conceivable that with more number of the heteroatom containing surfactant (1), the microenvironment is 
relatively polar, thus populating the micelle with more number of surfactants $\mathbf{5}$, which contains only hydrocarbon tail, causes the increased hydrophobicity of the microenvironment around the dye molecules inside the reorganized micelle as reflected in the blue shift in their emission spectra. Moreover, the insertion of the cleaved alkyl chain as a co-guest (see the hypothesis made in the main text) may also contribute to the increased hydrophobicity of the interior of the reorganized micelle for the residual amount of the dye molecule that might be left behind. It is reasonable to expect that the dye molecules do experience more than two different environments, because the change in the dye environment in this case is a continuous process. Each time disulfidecontaining surfactant is cleaved, the relative population of the two surfactants is changed in the medium and so is the microenvironment of the dye. Thus the blue shift is continuous.

Spectral data for dye release study from one of the mixed micelle $(1: 5=65: 35)$ at two different surfactant concentrations:
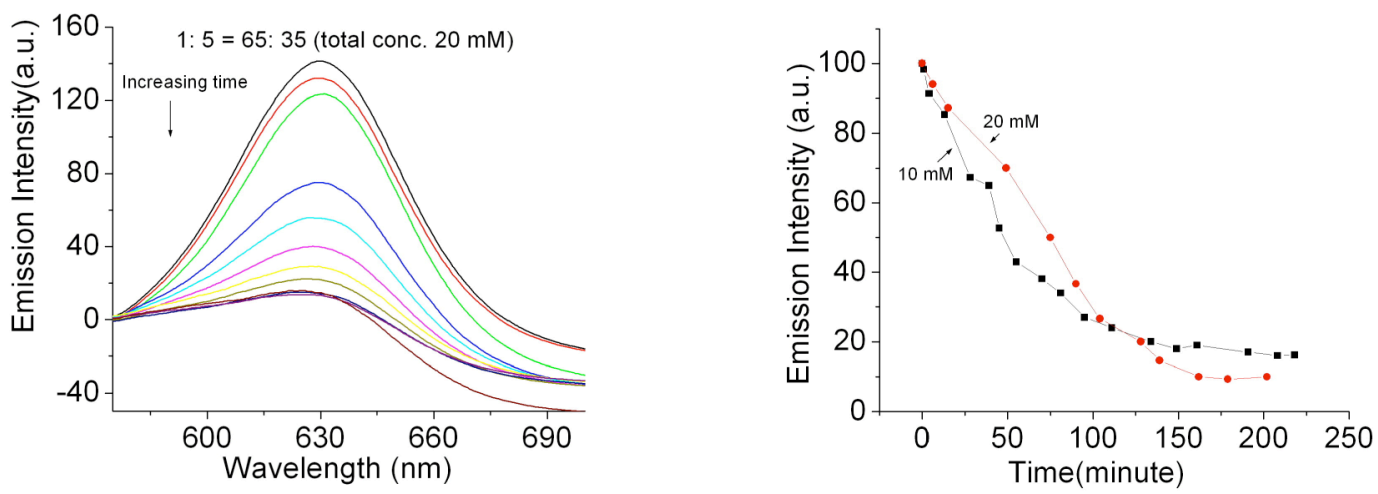

Figure S8: Dye release profile for mixed micelle $(1: 5=65: 35$, total surfactant concentration 20 $\mathrm{mM})$; left- the change in fluorescence spectrum with time, right-plot of fluorescence intensity (each time at emission maximum) against time and compare the data with that of the experiment done in $10 \mathrm{mM}$ concentration (Figure S4).

Experimental procedure for dye encapsulation studies using UV-Vis spectra: A stock solution of pyrene $\left(10^{-3} \mathrm{M}\right)$ was made in acetone. Measured amount of the stock solution was transferred to a vial such that when diluted to $2 \mathrm{~mL}$ the concentration of pyrene would be $5^{*} 10^{-5} \mathrm{M}$. Acetone was evaporated and $2 \mathrm{~mL}$ micellar solution $(10 \mathrm{mM})$ was added. The mixture was sonicated for 30 minutes and filtered. The UV-visible spectra were recorded from the filtered solution. Similarly the encapsulation of the Reichardt's dye (RD) was quantified for both surfactant $\mathbf{1}$ and $\mathbf{5}$ following identical experimental condition. 
Spectral data for relative encapsulation study of two hydrophobic dye molecule (Pyrene and Reichardt's dye) with the surfactants $\mathbf{1}$ and $\mathbf{5}$.
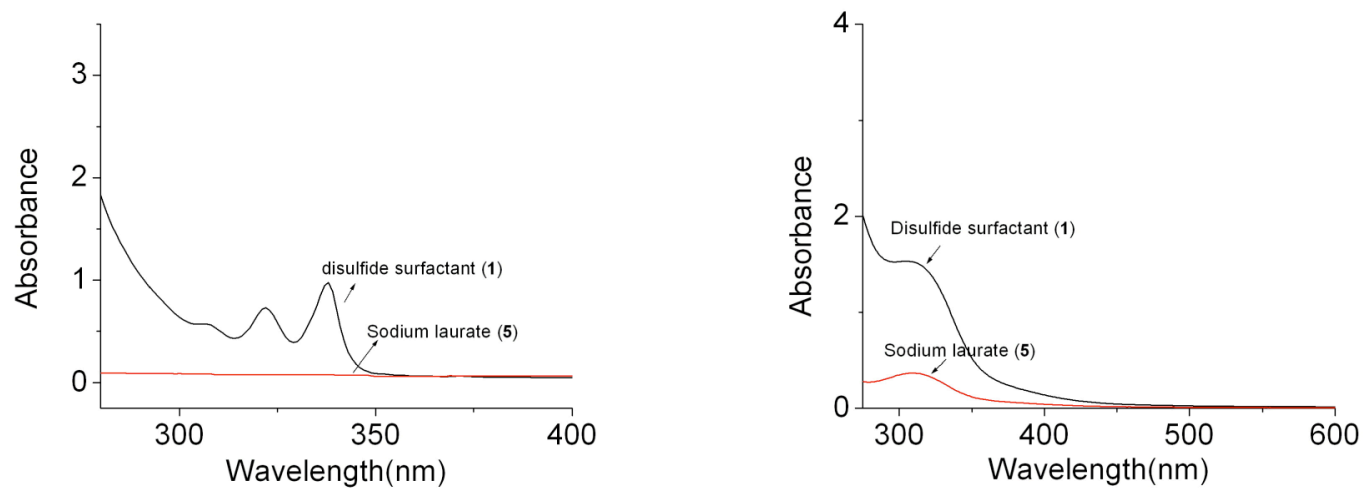

Figure S9: Absorption spectra of hydrophobic dye molecules in $\mathrm{H}_{2} \mathrm{O}$ in presence of surfactants $\mathrm{I}$ and 5; left-pyrene encapsulation, right-Richard's dye (RD) encapsulation. Concentration of surfactant in each experiment was kept constant at $10 \mathrm{mM}$.

Spectral data for dye release studies from micelle formed by surfactant 6 and comparison the rate with that of surfactant $\mathbf{1}$.
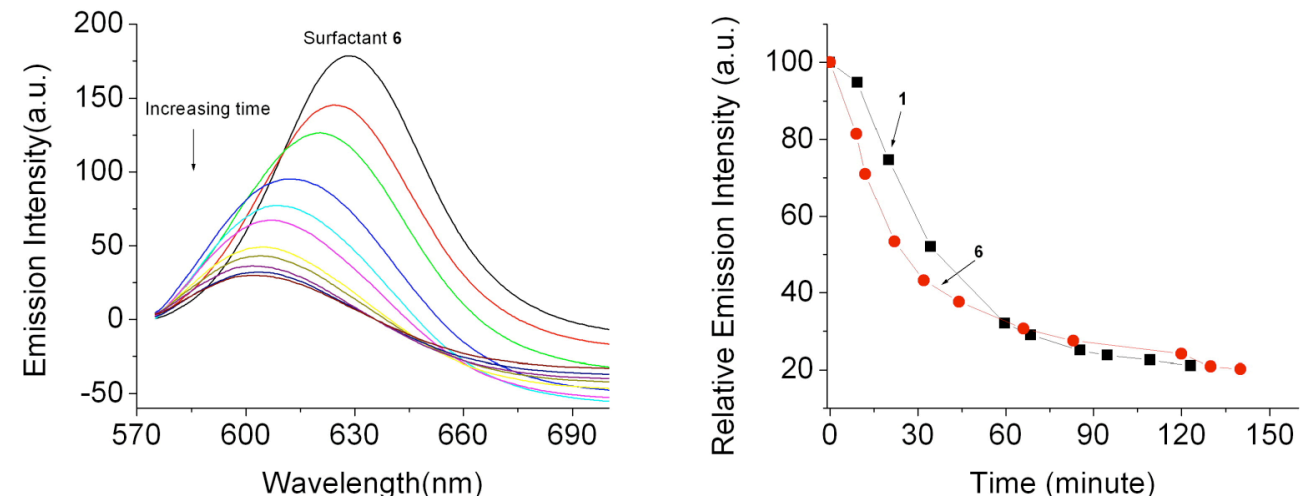

Figure S10: Dye release profile for micelle of surfactant 6; left-change in fluorescence spectra as a function of time, right- comparison of release profile between surfactant 1 and $\mathbf{6}$; Wave length (nm) at which the intensity is plotted for 6 (with increasing time) 634, 625, 620, 613, 607, 605, 604, 603, 602, 602, 601). For surfactant 1, the data has been copied from figure 1 in the main text. Total surfactant concentration $=10 \mathrm{mM}$, Concentration of Nile red encapsulated $1 * 10^{-5} \mathrm{M}$, and ratio of surfactant $1:$ DTT $=1: 1$ 


\section{Comparison between the excitaion and emission spectra of Nile red dye:}

Since there is a change in the emission spectra with cleavage, we searched for any differences between the absorption spectrum and excitation spectrum of Nile red under different cleavage conditions. This is included as an additional information about the system. We noticed a significant difference between the two spectra consistently for all the mixed micelle system as well as the micelle formed by only surfactant 1 . However, this difference appears to be due to the inherent properties of Nile red, as evidenced by the similar difference between the absorption and excitation spectra of this dye molecule in even solvents like acetone and dichloromethane where no aggregation should happen. We also have examined the excitation spectra as a function of wavelength and found it does not change with wavelength. The spectral data is provided below.
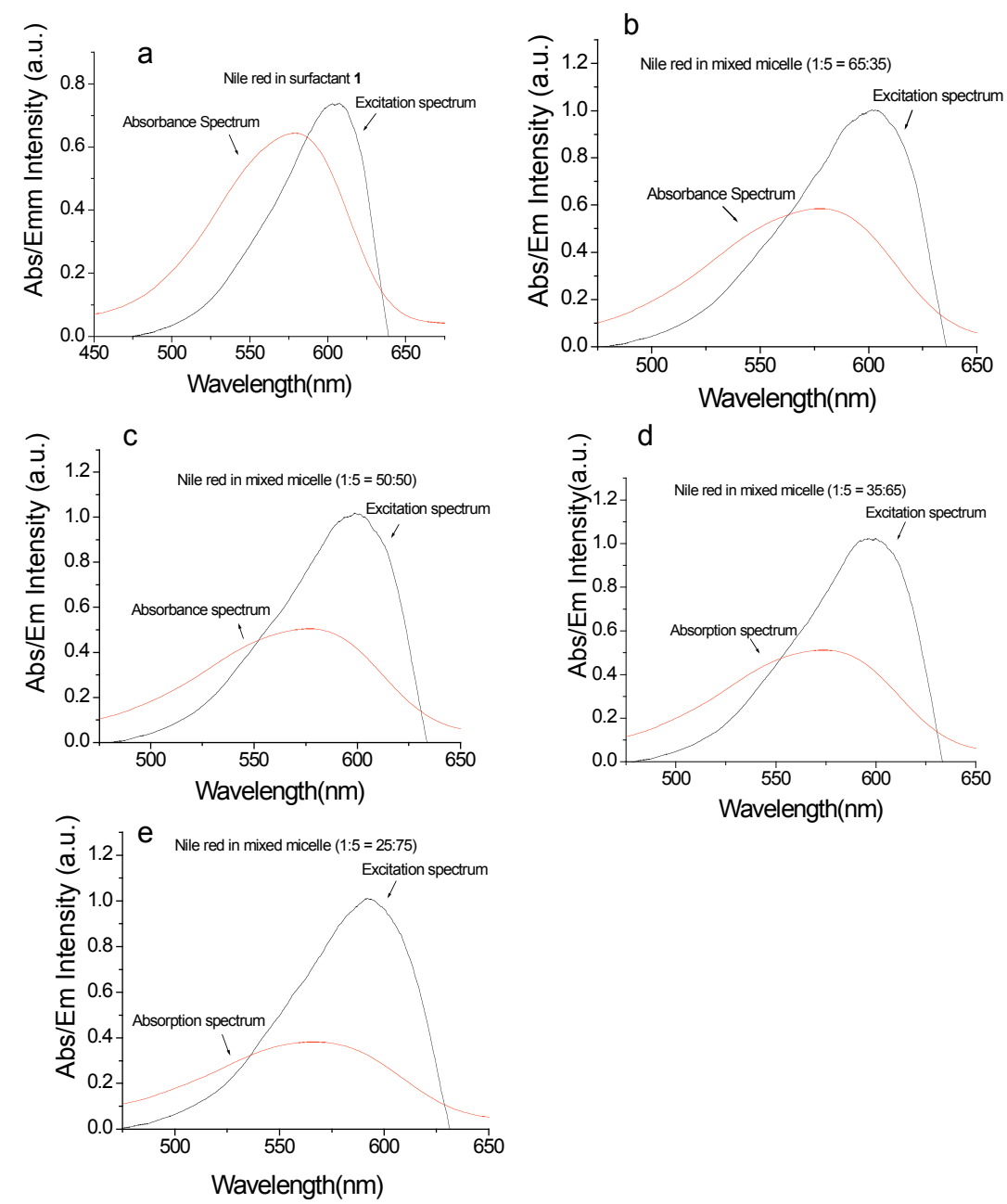

Figure S11: Normalized excitation and absorption spectra of Nile red in various mixed micelle (a-e); Nile red concentration is $10^{-5} \mathrm{M}$ in each experiment, surfactant concentration is $10 \mathrm{mM}$ for all the mixed micelle, excitation wavelength $=550 \mathrm{~nm}$, emission wavelength $=675 \mathrm{~nm}$ 

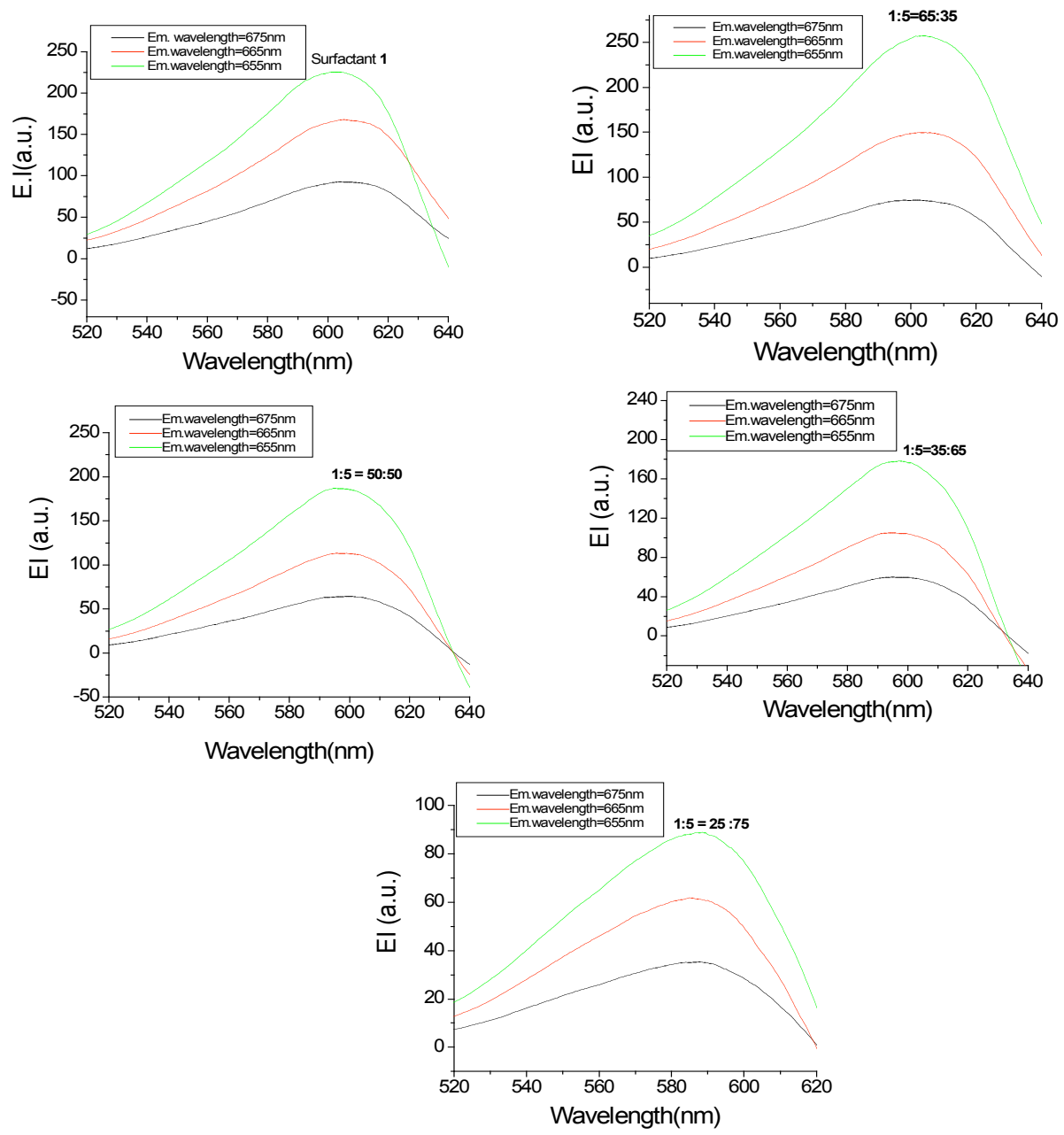

Figug12: Excitation spectra of Nile red, encapsulated in various mixed micelles in three different emission wave- length. Conc. of Nile red $10^{-5} \mathrm{M}$, total concentration of surfactant $10 \mathrm{mM}$. The emission wavelength and mixed micelle composition are mentioned inside each plot.
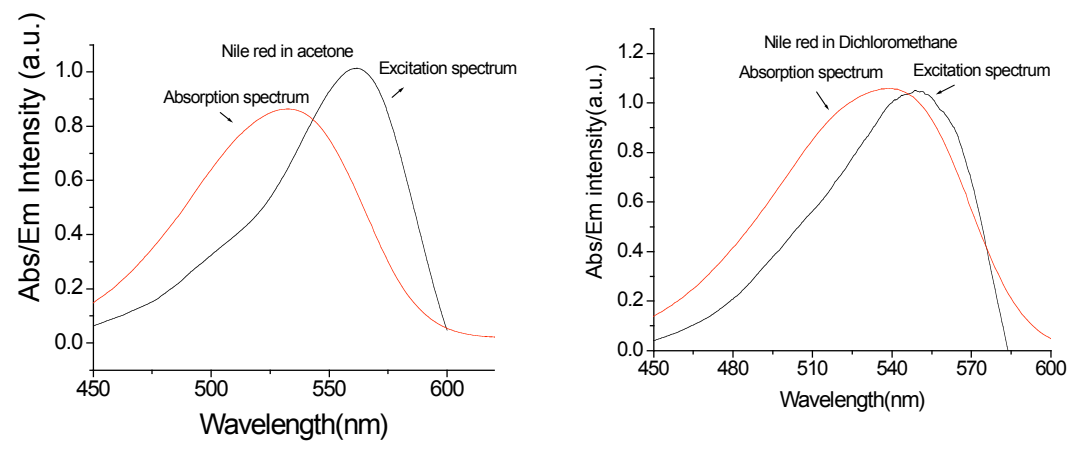

Figure S13: Normalized excitation and absorption spectra of Nile red in acetone (left) and dichloromethane (right): Nile red concentration is $10^{-5} \mathrm{M}$. Excitation wavelength $=550$ $\mathrm{nm}$, emission wavelength $=675 \mathrm{~nm}$ 


\section{Reproducibility of data for DTT induced release of Nile red from micelle of surfactant 1:}

In order to test the reproducibility of the data we have repeated the experiment and the comparison of the data in those two experiments are presented in Figure S14. It can be seen that the data is reproducible and the error in maximum release time is insignificant. The likelihood of error in the data reported is expected to be maximum, when the release is fast and thus the emission intensity of Nile red changes significantly within short period of time. Thus we chose surfactant 1 containing micelle as the system to examine the reproducibility of the data, because in this case the inherent rate of release was found to be the fastest among various other systems studied.

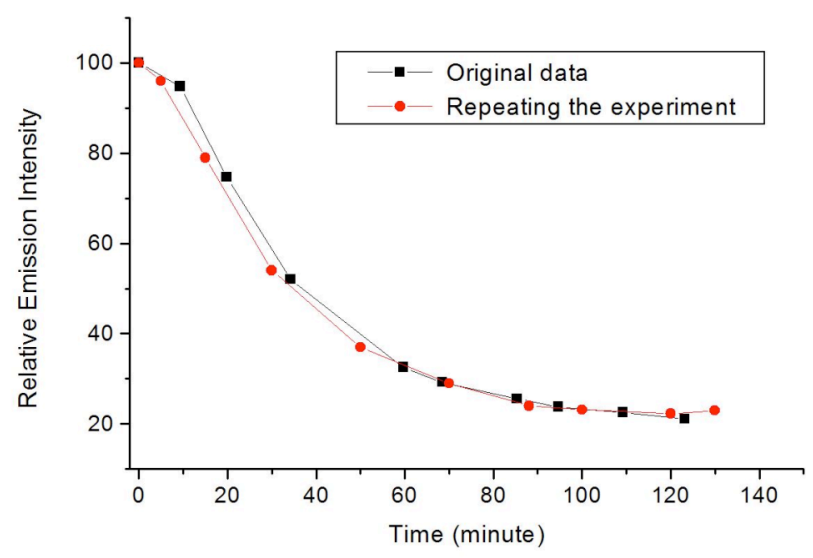

Figure S14: Comparison the data obtained for two experiments, performed under identical condition, for the change in fluorescence emission intensity of Nile red encapsulated in micelle formed by 1 in presence of DTT. In both the experiments the mole ratio of 1: DTT $=1: 1$ and the concentration of surfactant 1 is $10 \mathrm{mM}$. When the experiment was repeated, wavelength ( $\mathrm{nm}$ ) at which the intensity is plotted with increasing time is 631.5, 631, 629, 626, $620,619,614.5,614.5,615,614.5,615$. In the original experiment wavelength (nm) at which the intensity is plotted with increasing time is 631.5, 630, 629, 625, 621, 619, 614.5, 614.5, 614,615 